\title{
THEORETICAL STUDY OF PLASMA USING DIFFERENT DIMENSION OF HOLLOW CATHODE
}

\section{N. Mishra and K. Khanal}

Journal of Nepal Physical Society

Volume 5, Issue 1, October 2019

ISSN: 2392-473X

Editors:

Dr. Vinaya Kumar Jha

Dr. Binod Adhikari

Dr. Kapil Adhikari

JNPS, 5 (1), 30-34 (2019)

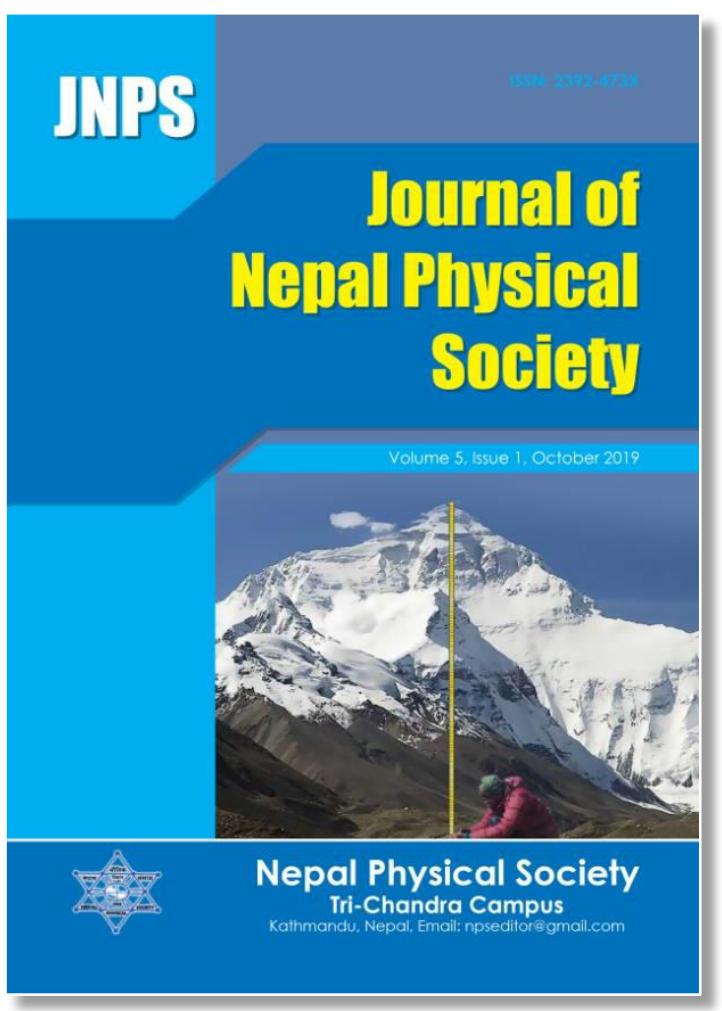

Published by:

Nepal Physical Society

P.O. Box: 2934

Tri-Chandra Campus

Kathmandu, Nepal

Email:npseditor@gmail.com 


\title{
THEORETICAL STUDY OF PLASMA USING DIFFERENT DIMENSION OF HOLLOW CATHODE
}

\author{
L. N. Mishra* and K. Khanal \\ Department of Physics, Patan Multiple Campus, Tribhuvan University, Lalitpur, Nepal \\ *Corresponding Email: Imisira@gmail.com
}

\begin{abstract}
This article deals about the theoretical study on DC hollow cathode glow discharge using different hollow cathode geometry. The mechanism of discharge is analyzed at various gas pressure and radial configuration. The dependence of temperature on gas pressure has been elucidated with the help of Schottky limit. Discharge behavior with radius has also been explained. It is revealed that floating potential increases as gas pressure increases whereas plasma potential decreases as gas pressure increases. This theoretical work resembles with the experimentally measured results. This work might be useful for the plasma processing for industrial purposes.
\end{abstract}

Key words : DC glow, discharge, Schottky limit, floating potential, plasma potentaial.

\section{INTRODUCTION}

The interest in the study of the hollow cathode discharge carried out throughout the decades seems to be influenced by its diverse applications such as electrical lamps, thin film deposition, sputtering, plasma display device and so on [1-8]. Hollow cathode discharge was first described by Paschen in 1916. Basically, a hollow cathode effect in glow discharges is the increase in the current density by several order of magnitude at an almost constant discharge potential. It is generally believed that these are due to the efficient geometrical confinement of ions, protons and metastable excited atoms in causing secondary electron emission from the cathode [9].

An experimental investigation of a DC hollow cathode nitrogen glow discharge has been done for different gas pressure ranged from 0.02 to 0.8 Torr and different radial position of discharge [10]. Hollow cathode discharges are capable of generating dense plasma and have been used for the development of high-rate, low-pressure, highefficiency processing machines. This geometric feature of hollow cathode discharge promotes oscillation of hot electrons inside the cathode, and thereby enhancing ionization, ion bombardment of inner walls and other subsequent processes, [11, 12]. Owing to its capability in achieving high current density in a short time, the pulsed hollow cathode discharge is suited for application as a high- power switch. Used as a high current closing
[13, 14], high current density with unheated cathode was attainable without the usual erosion associated with an arc, and, therefore, can have greater lifetime than spark gap under similar conditions. The role of the hollow cathode on the breakdown mechanism as well as the electron beam formation in the initial stage of the pseudo-spark has been much studied. The hollow cathode effect several mechanisms have been suggested to contribute to the hollow cathode effect phenomenon. They are as follows: (i) Electrons emitted from the cathode surface inside the hollow cathode structure which are accelerated in the cathode fall mainly contribute to ionization in the weak field region of the negative glow [15-17]. (ii) The cathode dark space in the hollow cathode under high current density conditions can be significantly thinner than in a planar cathode, reducing the probability for charge transfer collisions. Therefore, the average ion velocity at the cathode surface is increased, causing an increased secondary electron emission rate. (iii) A more efficient geometric confinement of the energetic neutral particles such as photons and metastable as well as the positive ions in causing secondary emission of electrons from the surface of the cathode. Thus a hollow cathode with larger ratio of the length or depth to its diameter or aperture would be able to trap these particles more efficiently increasing the probability of hitting the surface of the cathode. However, too large a ratio would introduce significant axial and 
radial inhomogeneity to some of the characteristics within the hollow cathode cavity. (iv) The higher plasma density inside the hollow cathode makes multi-step processes more likely. (v) The confined geometry of the hollow cathode leads to a higher density of sputtered atoms from the cathode material with lower ionization potential which influence the secondary processes at the cathode and in the volume of the discharge [18-21]. Penning ionization can also occur in rare gas hollow cathode discharge.

The basic design of the hollow cathode is shown in FiG1. having internal cathode length 1 and a diameter 2R. The backside of the cylinder can either be a cathode or another anode, depending on the application but, providing 1 and $2 \mathrm{R}$, the operation is similar. This follows because the cathode sheath is confined to a narrow layer between the cathode cylinder and the slightly smaller plasma cylinder. The anode can also have a central hole or be remote, depending on the application. Hollow cathode discharges have been operated at pressures of 0.1-10 Torr and current densities in the range of $0.01-1 \mathrm{~A} / \mathrm{cm} 2$ in tubes with $2 \mathrm{R}$ is $1 \mathrm{~cm}$ for use in atom and ion lasers [20].

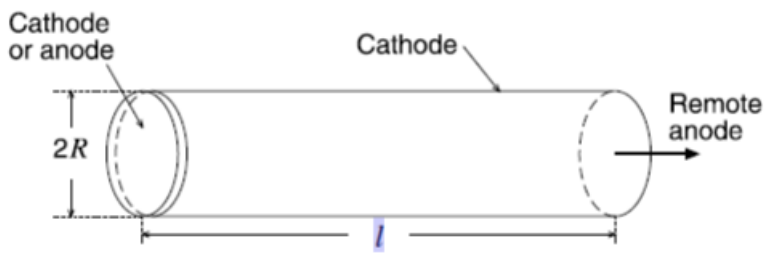

Fig.1: Cylindrical configuration of a hollow cathode discharge [20].

\section{THEORY}

This is based on the cylindrical equilibrium condition as in Fig. 1 where a discharge contains hot electrons, cold electrons, and positive ions with a cylindrical cathode having length ' $\mathrm{l}$ ' and radius ' $\mathrm{R}$ ' carrying a (radial) current $I_{d c}$ has been considered. The population of hot electrons is created by secondary emission due to ion bombardment of the two plates. The electrons accelerate across the sheath potential $V_{d c}$ and are trapped within the discharge by the confining space potential as a result the discharge is sustained. We assume that the pressure is low, such that the hot electron energy relaxation length $\lambda_{\varepsilon h}>2 R$ due to which hot electrons traverse the plasma before losing their energy to the background gas. If we consider a single positive ion species, then the plasma diffusion equation [20] is of the form $\frac{d^{2} n}{d r^{2}}+\frac{1}{r} \frac{d n}{d r}+\frac{G_{0}}{D_{a}}=0$

This can also be written as

$-D_{a} \frac{1}{r} \frac{d}{d r}\left(r \frac{d n_{i}}{d r}\right)=K_{i z} n_{g} n_{h 0}$

where, $n_{\mathrm{h} 0_{-}}$is the density of hot electrons generated by secondary emission from the cathode, $\mathrm{k}_{\mathrm{iz}}$ is the ionization rate coefficient for hot electrons with average energy $\sim V_{d c} / 2$ and $D_{a}$ is the ambipolar diffusion coefficient. This $\mathrm{D}_{\mathrm{a}}$ depends on the cold electron temperature, which is determined by a power balance relation for cold electrons.

\section{INFLUENCING PARAMETERS AND THEIR GOVERNING EQUATIONS}

This section describes the different parameters which affects the discharge the gas as well as their theoretical derivations with suitable assumptions.

Electron Temperature as function of pressureradius product:

Electron temperature is an important parameter in the positive column of a gas discharge. Once the electron temperature is known, a good estimate can be made of all the other parameter such as plasma electron density, ion density and so on. In this case, electron temperature $T_{e}$ can evaluated with the plasma balanced equation in terms of pressure and radius called pressure -radius product that is $\mathrm{pR}$, where $\mathrm{p}$ is the neutral gas pressure and $\mathrm{R}$ is the tube radius. Moreover, Schottky limit is employed in plasma balanced equation to generate theories covering low- and high-pressure discharges. The well-known Schottky solution [21] is

$$
\frac{\exp \left(e V_{i} / k T e\right)}{\left(e V_{i} / k T e\right)}=1.16 \times 10^{7}(c p R)^{2}
$$

where $\mathrm{V}_{\mathrm{i}}$ is the ionization potential in $\mathrm{V}, \mathrm{c}$ is a constant that depends on the type of gas and the product $\mathrm{pR}$ is measured in Torr $\mathrm{cm}$. Since (3.1) is given by an implicit function of the similarity variable $p R$, the temperature $T$, is usually obtained graphically. It is the purpose of this paper to find a simple relation which expresses $\mathrm{T}$, as an explicit function of $\mathrm{pR}$.

\section{Plasma Potential, $\boldsymbol{V}_{\boldsymbol{p}}$}

For Maxwell-Boltzmann distribution, current collected by the probe is given by

$$
I=\frac{e n v_{t h} A}{4} \exp \left(e\left(\frac{v-v_{p}}{k_{B} T}\right)\right)
$$


When $\mathrm{V}>V_{p}$, the electron current to the probe continues to grow, but only because of expanding collecting area due to an expanding plasma sheath. The boundary between two cases is defined by the point of maximum slope. The point where the slope is a maximum, of course, has the second derivative zero an inflection point. Thus $V_{p}$ is defined by I " $\left(V_{p}\right)=0$.

\section{Floating Potential and Plasma Potential}

The floating potential is defined by $\mathrm{I}\left(V_{f}\right)=0$. Of course, it is unlikely that any collected data point exactly has $I=0$. Instead a sequence of points with $\mathrm{I}<0$ will be followed by points with $\mathrm{I}>0$, with $V_{f}$ lying somewhere between two measured points. We seek to interpolate to find the best estimate for $V_{f}$.

\section{RESULTS AND DISCUSSIONS}

This section explains about the result and discussion. Graphs are depicted in the figures with the help of Matlab under Schottky limit. The different parameters such as electron temperature, floating potential, plasma potential with pressure as well as discharge radius as follows.

The study of the variation of electron temperature with Gas pressure:

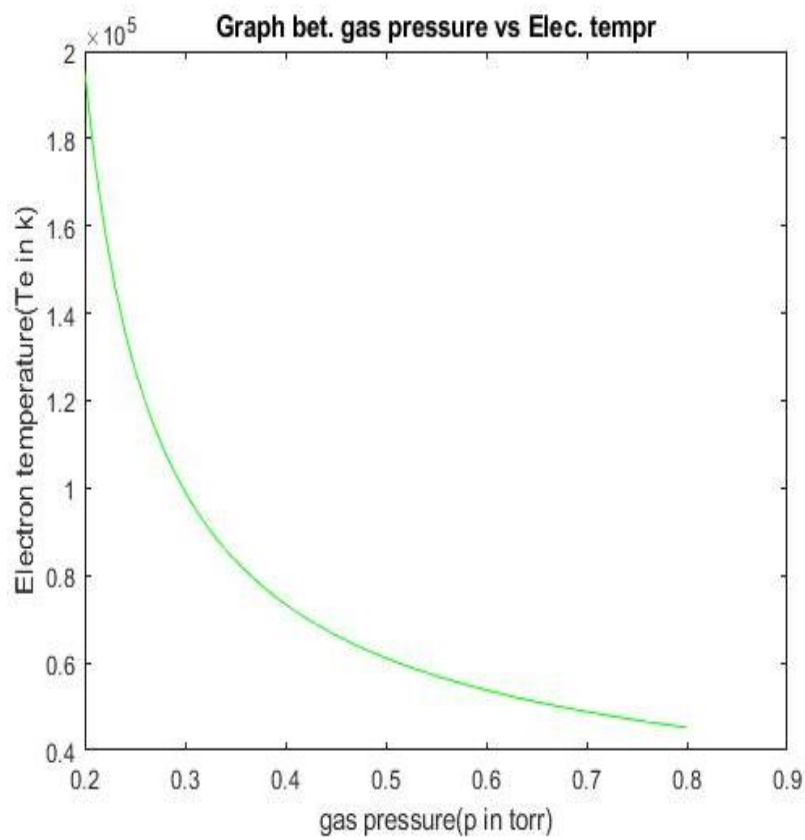

Fig. 4.1: The variation of electron temperature with gas pressure.

Figure 4.1 represents the variation of electron temperature with gas pressure. It explains that electron temperature varies with the change in gas pressure. In this case, gas pressure is taken as independent variable taken in $\mathrm{X}$-axis while the corresponding value of electron temperature as predicted by the reference equation is rendered as dependent variable taken in the Y-axis where electron temperature is found to be the order of $10^{5} \mathrm{~K}$ with variation in gas pressure in the range of 0.2-0.8 Torr. The graph clearly shows that electron temperature decreases exponentially with the rise in gas pressure. This kind of behavior can be attributed to the large mean free path of the electron at low pressure. And as a consequence, the electron, during its travel has low collision frequency with the other plasma particles and thus loose less energy.

\section{Graph between electron temperatures with} discharge radius:

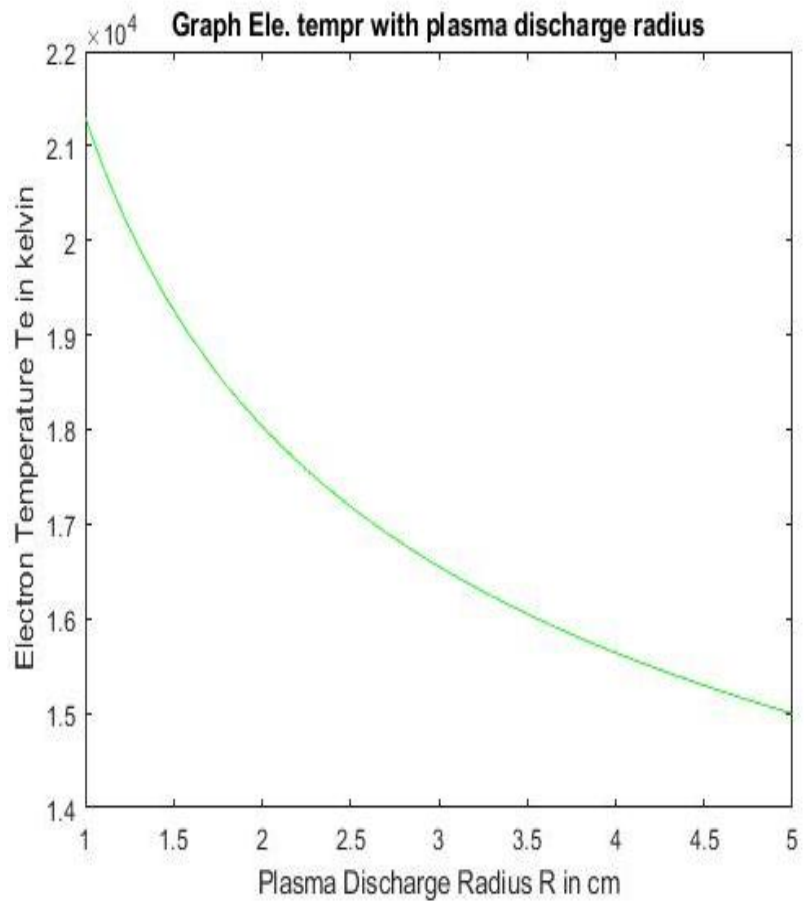

Fig. 4.2: The variation of electron temperature with discharge radius.

Figure 4.2 is the graphical representation between electron temperature and plasma discharge radius which depends on the geometry of the hollow cathode. It infers that plasma temperature decreases as plasma discharge radius increases. As the discharge radius increases, electrons diffuse to the wall promptly due to which temperature of electron reduces significantly. 


\section{Pressure with radius:}

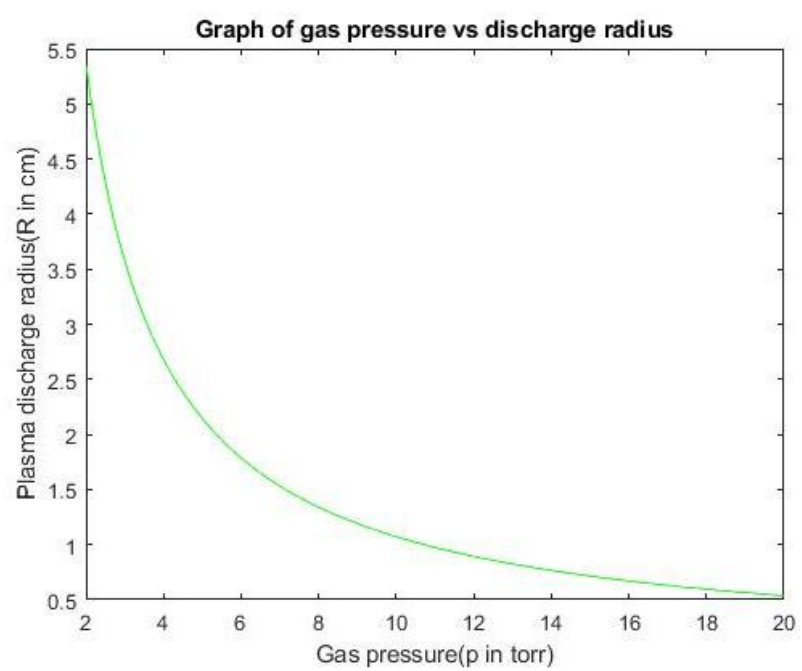

Fig. 4.3: The variation of plasma discharge radius with gas pressure.

Figure 4.3 is the graphical representation between plasma discharge radius with gas pressure. It infers that plasma discharge radius decreases as gas pressure increases. As the pressure increases, electrons collide with neutral gas molecules as a result plasma discharge radius decreases.

The study of the variation of Plasma potential with the change in the magnitude of gas pressure

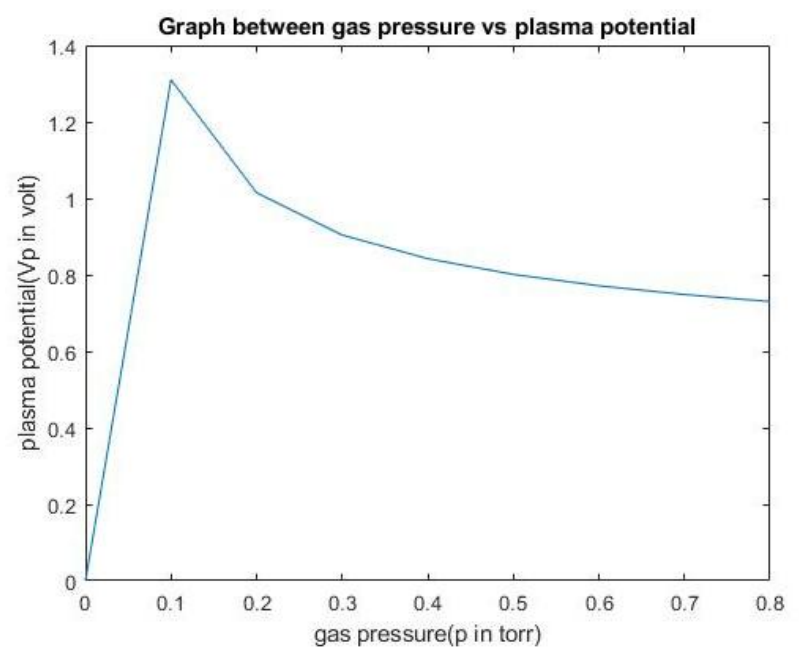

Fig. 4.4: The variation of plasma potential with gas pressure.

Figure 4.4 is the graphical representation between plasma potential with gas pressure. It is revealed that plasma potential increases slowly at first and then it will attain maximum value as gas pressure increases. After then it will decrease and attain constant potential as gas pressure increases further more.

\section{Graph between Gas pressures against floating potential}

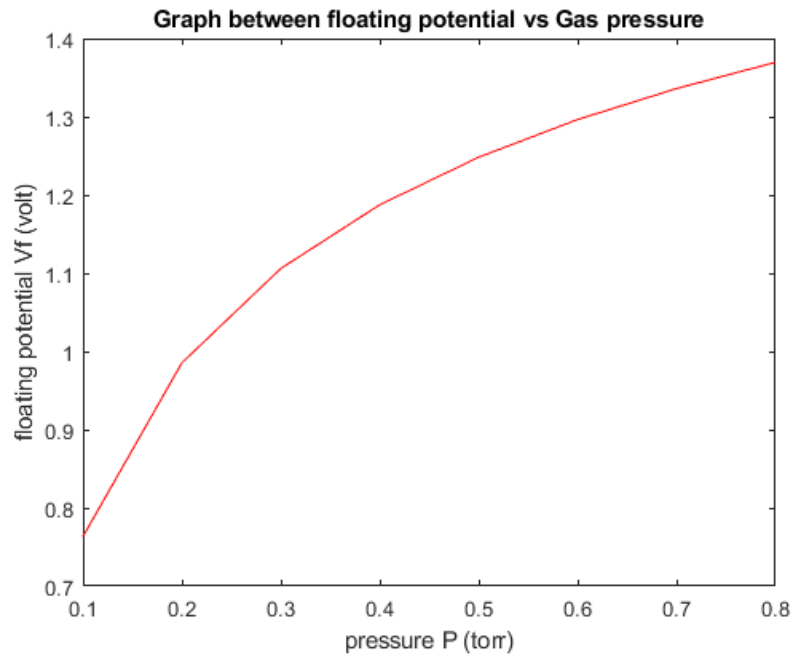

Fig. 4.5: The variation of floating potential with gas pressure.

Figure 4.5 is the graphical representation between floating potential with gas pressure. It shows that plasma potential increases as gas pressure increases.

\section{CONCLUSIONS}

This section concludes the theoretical study on the DC hollow cathode discharges where it can be realized that the properties of the hollow cathode discharge or the hollow cathode effect depends on a number of parameters which can be grouped into the hollow cathode geometry and the discharge operation conditions. It has further been brought to the attention that through many investigations on the hollow cathode discharge have been carried out, the parameters used and the properties studied are too varied, making comparison or correlation among them are not so easy task. The mechanism of discharge is analyzed at different gas pressure ranged and radial configuration. Plasma properties were inferred from the current-voltage characteristics at different radial space of the discharge. The effect of varying discharge with current has been characterized. The dependence of temperature on gas pressure has been elucidated with the help of Schottky limit. It is revealed that floating potential increases as gas pressure increases whereas plasma potential decreases as gas pressure increases. 


\section{REFERENCES}

[1] H. Kitagawa, A. Tsunoda, H. Shindo and Y. Horiike, "Etching characteristics in helicon wave plasma" Plasma source Science and Technology, 2,11 (1993).

[2] L. Woods, "Hydromagnetic waves in a cylindrical plasma,” J. Fluids Mech,13, 570 (1962).

[3] M.A. Lieberman, Principles of Plasma Discharge and Materials Processing, p. 2, Michael A. Lieberman (2003).

[4] H. Eichhorn, K. H. Schoenbach, and T. Tessnow, Paschen's law for a hollow cathode discharge, Physical Electronics Research Institute, Old Dominion University, Norfolk. Virginia 23508 (1992).

[5] A. Frederick Maxfied \& R.R Benedict "Theory of Gaseous Conduction and Electronics" McGraw Hill 1941.

[6] S.Yan, H. Kamal, J. Amundson and N. Hershkowitz, Use of Emissive Probes in High Pressure Plasma, Rev. Sci. Instrum. 67, 4130 (1996).

[7] J. Chen, S. Park, Z. Fan, J.G. Eden and Chang Liu (2002), 'Development and characteristics of micro machined hollow cathode plasma display devices" 11, 536 ( 2002).

[8] T. Kaneda, "The plasma parameter of a positive column in a transverse magnetic field" J. Phys. D: Appl. Phys. 12, 61, (1979).

[9] D. Akbar, The Non-Uniform Argon DC Glow Discharge Plasma System Parameters Measured with Fast Three Couples of Double, $33^{\text {rd }}$ EPS Conference on Plasma Phys. Rome, 19 - 23 June, 30I, D-5.010 (2006).
[10] F.F. Chen and R.W. Boswell, IEEE Trans. Power Appar Syst. 25, 124 (1997).

[11] E. Applentom, J.inst. Electr. Eng. 71, 642 (1932).

[12] J.P. Klosenberg, B. McNamara, and P.C. Thonemann, J. Fluid. Mech, 65, 545(1965).

[13] R.W. Boswell, and R.K., Porteus," "Large volume, high density $\mathrm{rf}$ inductively coupled plasma” Appl. Phys, Lett., 50,1130 (1987).

[14] M.A Liberman and Gottscho, Physics of Thin film, 18, New York (1994).

[15] C. Charles, "Ion energy distribution function in a multipole confined argon plasma diffusing from 13.56-MHz helicon sources," J. Vac Sci. Technol. A, 11,157 (1993).

[16] F. Chen, "Excitation of large amplitude plasma waves", Physica Scripta T, 30,14 (1990).

[17] M.J. Druyvesteyn and F.M.Penning, "Mechanism of electric discharges in gases of low pressure" Rev. Mod. Phys.12,87 (1940).

[18] F. F. Chen, Introduction to plasma physics and Controlled Fusion, Second Edition, Plenum Press, New York (1984).

[19] F. E. Rose, M.T. Taylor and R. Boswers, "Low frequency magneto-plasma resonances in sodium" Rev,127,1122 (1962).

[20] M.A. Lieberman, A.J. Lichtenberrg, Principles of Plasma Discharge and Materials Processing, Second Edition, p. 551, A John Wiley and Sons, Inc. Publication (2005)

[21] M. Sato, "Electron Temperature as an Explicit Function of Pressure-Radius Product in the Schottky Theory", J. Phys. D: Appl. Phys. 67, 4130 (1989). 